\title{
APORTES DE LA GEOMETRIA DE COMIENZOS DEL SIGLO XIX A LA TRANSFORMACIÓN DE LAS MATEMÁTICAS
}

MARIo H. OtERo

1. El título que hemos elegido, el del aporte de la geometría de comienzos del siglo xIx a la transformación de las matemáticas, contiene ciertos presupuestos que de hecho apuntan a problemas delicados, nada simples.

1. Transformación, ¿qué quiere decir? Por lo menos dos problemas se plantean: transformación, ¿en qué?; 2. Transformación, ¿de qué?

Para responder estas preguntas rápidamente podríamos decir: ¿En qué? En la geometría de los Grundlagen de Hilbert. ¿De qué? Por lo menos de dos cosas:

a) del discurso geométrico mismo,

b) de ciertos supuestos de ese discurso geométrico que sólo a veces aparecen traducidos en el discurso mismo. Cuando se ha hablado reiteradamente de esa transformación, a menudo se ha apuntado sólo a este segundo aspecto. Pero no siempre. En todo caso no ha habido un esfuerzo constante para distinguirlos.

1.1. Vayamos algo más despacio. Respecto al primer asunto (¿en qué?), supongamos por el momento que los Grundlagen constituyen el hito que recoge los cambios efectuados con anterioridad, en, por lo menos, todo el siglo anterior. $Y$, además, que la matemática subsiguiente por un buen periodo (¿hasta hoy?) comparte las características de esa obra o las acentúa.

Responder de ese modo plantea ya bastantes cuestiones nada sencillas, pero hay una, general, que surge siempre. Cuando se hace historia de la ciencia, ¿estamos autorizados a proceder desde el presente hacia atrás, o ello compromete los resultados? Podría afirmarse que de procederse así no se hace historia de la ciencia sino otra cosa (aunque ésta no es una opinión universalmente compartida, como bien sabemos). Según aquel punto de vista, colocar el presente (o un presente-pasado, por ejemplo Hilbert-99) como término de la historia conllevaría desconocer los laberintos y callejones sin salida de la historia real y además inundaría el discurso con una teleología inaceptable. Frente a ello se afirma que toda producción historiográfica se hace desde cierta perspectiva, que toda ex- 
plicación comporta modelos explicativos actuales. $Y$ que, además, teleología es apenas un término peyorativo para una actividad historiográfica de todos modos controlable y que posee su objetividad. Se trata de una polémica muy conocida, importante y que de ningún modo quiero malbaratar de pasada.

El caso es que lo que hoy hacemos (llámese historia de la ciencia o con otro nombre) no se sitúa al extremo opuesto de la intención del quehacer historiográfico; y que introducir el término "transformación" implica siempre ese gran problema. Es evidente que lo ha implicado en gran parte del discurso producido sobre... la transformación de la matemática, y de tantas otras cosas y procesos, científicos o no.

No quiero desconocer que existe el problema, pero creo que de cualquier modo es lícito plantearse cierto tipo de preguntas.

Por otra parte, el hecho de que el hito que se fija, los Grundlagen, sea el de un presente-pasado ya bien pasado, que se posea un distanciamiento significativo que no existiría de tomarse un hito de otro tipo (digamos el primer número de 1982 de Mathematical Review o los Elementos de Bourbaki, para tomar casos nada ficticios, y que han dado lugar a dos libros de historiografía bien conocidos y discutidos aún más), el hecho de que se posee además un conjunto de trabajos historiográficos de detalle durante un periodo de 80 años tampoco es un elemento desdeñable.

De cualquier modo, puede decirse que tomar ese hito Hilbert-99 (como término de la transformación apuntada) no implica para nada adoptar la filosofía formalista que Hilbert desarrolló, sino las características (y ciertos supuestos, discutibles con buenas razones) de los Grundlagen. No es tampoco pensar que Poncelet o Steiner deban ser Hilbert avant la lettre (o avant l'axiomatique). Por otra parte, plantearse que durante un buen periodo la matemática compartió las características de los Grundlagen y las acentuó no es para nada pensar que la matemática hoy sea sólo eso.

El problema a que nos referimos no es traído por los pelos. Lo han planteado diversos autores y entre ellos dos a quienes quiero referir muy brevemente: Lakatos en sus Proofs and Refutations (1976, pero de elaboración bastante anterior) y Giorgio Israel en un artículo reciente (1980).

Si la matemática de hoy es otra cosa que Hilbert-99 no es lo que está en cuestión. Por lo señalado antes: el presente-pasado del hito fijado. Si es algo diferente lo es asumiendo además el modo de los Grundlagen.

Si, como Israel o como F. E. Browder (1975), se piensa que la matemática es efectivamente otra cosa que desarrollar la filosofía formalista o aun el modo Hilbert-99, no es de todos modos suprimiendo lo que la 
axiomática aportó que los matemáticos trabajan hoy. Es un tema grande. Baste con decir esto: En cuanto a la historiografía misma, o a una tarea perihistoriográfica para nada inconsistente con ella, sigue teniendo sentido preguntarse: ¿cómo se transformó la geometria hasta Hilbert?

Aparte de las objeciones que despertó el planteamiento de Lakatos, podemos de todos modos pensar como lícito un planteamiento del tipo que aquí intentamos. De cualquier modo, vale là pena en algún otro lugar discutir detalladamente los argumentos de Israel y similares sobre los condicionamientos de la producción historiográfica referente a las matemáticas; no lo negamos, sólo lo postergamos.

1.2. Veamos, muy brevemente también, el segundo aspecto. La transformación, ¿de qué? ¿Del discurso geométrico en sí mismo o de ciertos supuestos suyos que sólo a veces aparecen traducidos en el discurso mismo? ¿De ambas cosas y de nada más?

Hay autores que han llegado a afirmar (es el caso de Crowe) que sólo del discuro geométrico se trata (o del discurso matemático). Sobre la base de esa premisa y de una restrictiva definición de revolución para el caso de las matemáticas se concluye, casi como en un teorema, que no ha habido revoluciones en las matemáticas. No es el caso discutir aquí si las hubo o en qué sentido las hubo.

Pero bastaría extremar las cosas (y se lo podría hacer con sentido nominalista, que sus dificultades posee) para que la historiografía fuera sólo de las manchas de tinta sobre el papel de ciertas cosas que los bibliotecarios han clasificado como revistas o libros matemáticos. La historiografía resultante reduciría, hasta casi anular, su objeto.

Pensamos por el contrario que cuando Nagel (1939), entre otros, muestra cómo va diluyéndose el espacio o la extensión como objeto de la geometría, ésa no es una afirmación carente de sentido por el hecho de que la palabra "extensión" no aparezca en ciertas obras de geometría que visiblemente suponen un correlato físico para sus enunciados. El debilitamiento de la geometría física, y sus avatares, sería, para una posición de ese tipo, algo totalmente ajeno a la historia de la geometría. Pero no es así. Ni siquiera sólo el discurso geométrico y sus supuestos agotan el material de la historia de la geometría. Para el siglo xIX no es indiferente, por ejemplo, que la difusión de la producción geométrica esté sujeta a una serie de vicisitudes que poseen sus efectos nada escasos.

Se tomen como obvios o como discutibles, éstos son algunos de los puntos que supone nuestro tratamiento del tema; no se trata de supuestos soslayables ni de elementos que se encuentren por encima de toda sospecha. Pero consideré necesario plantearlos de entrada, antes que nada. Con esto se abren problemas, no se cierra ninguno y se puede trabajar. 
2. Cuando hablamos del proceso de transformación de la matemática que culmina con Hilbert (1899) siguen siendo problemas (respecto a la geometría) por lo menos dos cosas:

1. ¿Cómo se desarrolló el proceso y cuáles fueron sus principales hitos?

2. ¿En qué sentido los Grundlagen son culminación de un proceso o sólo una expresión más, aunque extremadamente significativa, del mismo?

2.1. Ha sido muy repetida la boutade de Russell en el sentido de que la matemática pura ha sido bautizada mucho antes de nacer y que sólo Boole ha sido su alumbrador. ¿No estaba exagerando Russell, en un sentido distinto al que se propuso, dando una fecha prematura? (Su frase es de 1901.) Se ha hablado mucho del proceso indicado, se lo ha analizado con detalle; pero, ieste mismo abigarramiento de detalles no ha sido el bosque que ha impedido ver claro, hasta hoy, las fechas claves, o lo que ha hecho oscilar mareadoramente las propuestas de momentos decisivos? El trabajo de elaboración historiográfica del periodo está en pleno desarrollo hoy. Sólo pretendemos encarar aquí un punto (el aporte de la geometría proyectiva); pero de un modo u otro, una decisión en ese punto supone una interpretación del conjunto del proceso.

2.2. Cuando decimos que el proceso culmina con Hilbert estamos utilizando una hipótesis más que razonable (apoyada por elementos nada desdeñables): la de que en los Grundlagen se da un conjunto de características paradigmáticas (que no especificaremos por el momento), que van a ser, como dijimos antes, las de la matemática posterior por un buen periodo. Pero, ¿'son los Grundlagen una culminación o sólo un momento?

Si atendemos a lo que dice el mismo Russell en 1897 o a la resistencia que Frege opone a la manera hilbertiana de construir la geometría, podríamos pensar que, por más que la geometría de Hilbert sea para nosotros un momento cumbre, estaba lejos de ser su manera de ver una dominante en el borde de los dos siglos.

Creemos que se imponen dos constataciones:

Los hitos del proceso de transformación de la geometría no han sido todavía suficientemente esclarecidos. Por más que el Hilbert de 1899 sea paradigmático (se trata de una hipótesis apoyada por elementos de prueba indudables), la concepción de la geometría correpondiente no estaba todavía impuesta en ese momento o por lo menos no lo estaba en el pensamiento de destacados filósofos de la geometría (y destacadísimos científicos), como lo fueron Frege y Russell. Pues el kantismo sobrevivía a sus mismas cenizas. Es cierto que podría pensarse al respecto en un décalage entre geometría y filosofía de la geometría, y afirmar un retraso de la segunda respecto al desarrollo de la primera. Nos inclinamos en ese sentido. Pero de todos modos es necesario tener clara la diferencia 
entre esos dos tipos de dominancia, para, con pleno sentido, poder indicar cuándo en uno y otro aspecto se produce un giro fundamental o giros significativos. En geometria, aunque no necesariamente en la filosofía que de ella se difunda, estaría pues ya en Hilbert-99 "sancionada oficialmente" (Lolli) la transformación de la geometría, y ésta es nuestra primera hipótesis.

2.3. Una segunda hipótesis sobre el proceso que se cumple a lo largo del siglo XIx, la de que se dan una serie de hitos muy significativos que es necesario elucidar, podría parecer trivial. Sin embargo, ella se opone a la idea de una evolución más o menos continua y también a la de un único momento crucial en que se cumpliría, de golpe, el proceso a que nos estamos refiriendo.

Vamos a proponer un esquema extremadamente general que nos sirva de base para concretar esa segunda hipótesis, aunque todavía sin referencias temporales ni nominales. El cambio de una geometría física, de contenido, que presupone al espacio o la extensión como objeto de la disciplina, hacia otra en la cual las estructuras adquieren calidad temática, cambio en el cual los pasos deductivos en la construcción de la geometría logran una preponderancia sobre los momentos intuitivos, sería la línea básica del esquema.

Ese proceso, que podría llamarse abstracción creciente (a saltos), "lleva más tarde hacia" Hilbert-99, a través de una especial elaboración en tres aspectos: a) el rigor (muy claro en el desarrollo del análisis y del álgebra, pero también señalable en geometría), $b$ ) la fundamentación interteórica (entre ramas "distintas" de las matemáticas), y $c$ ) la axiomatización, con sus correlatos, comienzo de formalización por un lado, y aparición de elementos de análisis metamatemático, por otro. Cómo se dieron históricamente es otro asunto. Si bien esta visión es esquemática, y algo ingenua, sobre todo visto el desarrollo reciente de la historiografía, de todos modos puede servirnos de base como esquema plausible para considerar el tema específico del aporte de la geometría proyectiva de comienzos del siglo xrx al proceso total de desarrollo de la matemática.

3. El periodo principal que nos interesa se extiende desde 1822, fecha de publicación del Tratado de Poncelet (es decir la fecha del acabamiento de la primera fase de la geometría proyectiva) hasta 1847 , que marca el momento en que von Staudt produce una geometría no-métrica, como era la vocación de los desarrollos anteriores. Pero nos interesan también los periodos comprendidos entre esa fecha y 1867 , el de $1867-1872$, y en menor medida el subsiguiente hasta Hilbert, a efectos comparativos.

$Y$ dentro de ellos se dan fenómenos que podríamos llamar de producción inicial, de difusión, de trabajo elaborativo y de integración, a los 
que subyace la aludida transformación en el objeto de la geometría, que en definitiva queremos ver en qué consiste.

Durante el periodo que va desde 1829 hasta 1867 (la obra de Lobatchevski es de 1829 y la de Bolyai de 1832), las geometrías no euclidianas han sido constituidas inicialmente pero no han producido efectos significativos. Es bien sabido que la difusión de esas geometrías comienza alrededor de 1867 y difusión quiere decir constitución de una tradición sostenida de trabajo en las mismas. Las traducciones alemana, francesa (Houel), italiana e inglesa, y el trabajo capital en ese sentido de Beltrami, se sitúan apenas en ese momento, es decir casi cuarenta años después de la producción original, por causas que son hoy bastante bien conocidas y que resulta innecesario repetir. Si bien es cierto que posteriormente aparecen los trabajos fundamentales de Riemann, sólo la obra de difusión y elaboración de Helmholtz y los textos anteriormente señalados inician la tradición indicada. El trabajo propiamente dicho apenas comienza entonces (1867). Pero, habiendo pasado sólo unos cinco años más, Klein, en su programa de Erlangen (sobre la base del trabajo de 1859 de Cayley), produce ya la integración de las geometrías no-euclídeas con la geometría proyectiva, de modo que ambos tipos de geometría ya no poseerán desde ese momento desarrollos teóricamente independientes (por más que esto posea limitaciones en sus efectos). De modo que, como base plausible de comparación entre el trabajo independiente de la geometría proyectiva y el de las no euclidianas, deberían tomarse, con exclusión del trabajo de Riemann (que posee también efectos diferidos), lo producido en casi cuarenta años por un lado frente a apenas unos cinco por el otro. Esto, que podría aparecer grueso, se da sin embargo en sus grandes líneas así, por efecto de los fenómenos de difusión cientifica y de las condiciones de ésta. Entonces, con el fin de ver en qué medida la geometría proyectiva de la primera mitad del siglo contribuyó a la transformación de la matemática y en qué medida lo hicieron las geometrías no euclidianas, sería válido en principio considerar cuál fue el resultado de la producción geométrica en la primera durante justamente el casi medio siglo que va de 1822 a 1867; es decir, qué resultados logró, qué métodos utilizó, y cómo varió, en las propias obras cientificas, la concepción de la geometría.

Si pudiera constatarse que en ese periodo se produce un cambio (o cambios) muy significativo(s), habría que explicar, entre otras cosas, por qué la geometría proyectiva no tuvo sus beocios como los anunció Gauss, con visión predictiva adecuada, para las geometrías no-euclídeas. Los beocios no sólo actuaron como elemento de resistencia - extra-científica, filosófica fundamentalmente-, sino que contribuyeron en no poca medida a poner a las geometrías no euclídeas en el centro de la conside- 
ración epistemológica. Por otra parte Lotze y otros beocios sólo comenzaron a protestar tardíamente. Pero habría que ver además por qué entonces la geometría proyectiva no apareció como revolucionaria. Ver en especial si los elementos que ya habrán estallado en Hilbert-99 aparecen de un modo $u$ otro en aquella geometría proyectiva. $Y$ si esto parece exagerado, sería cuestión de ver cuáles de los elementos, aunque necesariamente no todos, de los que constituyen la gran transformación referi$\mathrm{da}$, se encuentran en forma implícita o patente en la geometría proyectiva del segundo cuarto del siglo entre Poncelet y Staudt. Pues resulta por demás curioso que la tradición epistemológica haya privilegiado retrospectivamente un momento del desarrollo de la geometría en detrimento de otro cuya significación se nos aparece hoy bajo nueva luz.

4. No voy a trazar aquí la historia de la geometría proyectiva en algunas líneas ni en muchas. Ello está bien hecho ya en sus aspectos fundamentales, aunque falta una puesta al día reciente: Desargues y Pascal, Monge y Brianchon, entre algunos otros, han aportado, decisivamente, los elementos de base en dos periodos distintos. Voy a dar por supuesto también que Poncelet, con su Tratado, que marca el principio del periodo que nos interesa (sobre todo 1822-1847), ha presentado magnificamente la disciplina en su conjunto. Voy en cambio a referirme puntualmente a algunos aspectos que resultan pertinentes para el tema del título. Los principales, a mi entender, son: el gradual surgimiento de métodos; en geometría, el uso de un nuevo lenguaje con su creatividad propia; la introducción del principio de dualidad y el carácter básicamente nométrico de la geometría proyectiva. Vayamos por partes.

4.I. Como es bien sabido, el periodo inicial, con Desargues como figura central, es sucedido por un siglo vacío. El dominio de la geometría analítica durante ese siglo es prácticamente total. Cuando la geometría proyectiva se constituye en disciplina, es la geometría sintética que renace, pero los procedimientos utilizados para ello son a veces analíticos, a veces sintéticos, a menudo mixtos, como los llamara luego Chasles. Aun la presentación fundamentalmente sintética de las meditaciones de Saratov recubre un conjunto de búsquedas analíticas, lo que no se revelaba en el Tratado, como bien surge de los Cuadernos de Poncelet, publicados con mucho retraso. Pero sea una u otra la vía de construcción, lo que predomina, lo que sucede es la búsqueda de métodos para la geometría sintética que superen el camino azaroso de descubrimiento típico de la geometría griega y tradicional, y que produzcan resultados de la manera en que justamente son producidos en geometría analítica.

Los métodos que van a utilizarse son variados, a veces muy discutidos como el principio de continuidad, o usados generalizadamente como el de 
polos y polares. De ese modo se trata, más que de enunciar teoremas aislados, de generar demostraciones de los mismos o de los que la tradición transmite; se trata de encontrar vías para hacerlo de una manera menos fortuita dentro de una disciplina que en principio poseía otros paradigmas. $Y$ no sólo se da en el caso de la racionalidad euclídea, de los métodos que hoy sabemos que tuvieron sus frutos y su trasmisión en el trabajo continuado, sino aun en otros que quedaron de lado por diferentes motivos; es el caso de los de la escuela napolitana de geometría y en especial de los métodos de Férgola.

En la geometría sintética, o de vocación sintética (recordemos a Steiner forzando la reconstrucción sintética pura), esta búsqueda de métodos de trabajo no es independiente de otras características determinantes. Es de por sí, como vemos, un síntoma de la influencia del modelo de construcción de la geometría analítica sobre una geometría que avanzaba de modo totalmente distinto.

4.2. El segundo aspecto a señalar posee una más larga historia. El uso de puntos impropios y de otros elementos impropios, historia (cuyas vicisitudes han sido estudiadas en su detalle), implica llamar 'punto' a algo diferente a lo que designaba con anterioridad esa palabra. La creación de lenguaje que ello importa, la generalidad que introduce y las consecuencias a que da lugar son facetas de la revolución incruenta, tranquila, poco espectacular, que implica toda la geometría proyectiva en su conjunto.

Ya la geometría analítica, geometría física si la hay, llamaba punto a un par ordenado de números, algo poco tradicional, y el hacerlo tenía consecuencias inesperadas. La ecuación de la recta permitía encarar intersecciones de rectas (mediante la solución de un sistema de ecuaciones), pero a la vez considerar otras cosas: rectas paralelas (por la solución imposible del sistema de ecuaciones correspondiente) y rectas coincidentes. El lenguaje así creado respondía por un lado a los nuevos conceptos introducidos pero, por otro, dio lugar a consecuencias no tradicionales, con sus efectos correspondientes.

Del mismo modo, los elementos impropios dan lugar, en la geometría proyectiva, a un lenguaje nuevo con un rendimiento teórico distinto al del lenguaje y al de los conceptos anteriores. Esto, que podría parecer trivial, constituye una característica determinante de las nuevas geometrías. En la geometría proyectiva todas las rectas se cortan en el plano, todos los planos se cortan en el espacio. Se trata de una geometría distinta, radicalmente distinta a la anterior. Por qué no apareció como revolucionaria, es un hecho por explicar. Sin embargo no todas las consecuencias de la nueva conceptuación y del nuevo lenguaje aparecen explícitas desde el comienzo. Avancemos desde ya la afirmación de que se 
trata en principio de una geometría no física (que ella tenga que ver con la perspectiva y sus leyes es otra historia): tanto la geometria de los Elementos euclídeos como la cartesiana eran físicas, y toda la geometría —salvo justamente la proyectiva - lo va a ser por un buen periodo.

4.3. El aspecto central que queremos encarar nuevamente aquí es el de la dualidad y sus consecuencias. Hay por lo menos tres temas de interés a su respecto que podrían encararse: 1) el del descubrimiento, 2) el de la demostración de un enunciado de la "ley" de dualidad, 3) el de su sentido. Me limitaré al tercero. Si bien por un lado la polémica entre Poncelet, Gergonne y Plücker es todo un tema (de precedencia en el descubrimiento), no lo voy a enfrentar aquí (Nuvoli). Si bien el tema de la demostración ha dado lugar a un conjunto de desarrollos bien interesantes, tampoco es ése nuestro objeto.

Vamos a dar por supuesto que Poncelet hace uso de la dualidad a propósito de un conjunto de propiedades, con base en la relación entre polos y polares (es decir, en una forma particular de relaciones); que Gergonne enuncia un principio de dualidad de modo general (introduciendo la escritura de columnas paralelas), atribuyéndole al principio un status a priori, y sin preocuparse por la demostración de sus propuestas generales.

La gradual introducción de elementos impropios, el uso de la polaridad, la relativa prescindencia de aspectos métricos y el estudio de los poliedros (la discusión de la propiedad de Euler) dan lugar a la aparición entre 1810 y 1830 del concepto de dualidad en la geometría proyectiva moderna. Me parece determinante de esta introducción lo que hemos llamado nueva conceptuación y, por consiguiente, la creación de un nuevo lenguaje. El rendimiento teórico de ambos se expresa entonces en la dualidad de la geometría proyectiva y en sus consecuencias.

4.3.1. El principio de dualidad, como "verdad absoluta", encierra dos proposiciones.

"En géometrie plane, lorsqu'un théorème indépendant de toutes relations métriques d'angles et de longueurs se trouve démontré, on peut conclure immédiatement, et sans qu'il soit besoin de démonstration, un autre théorème, dans lequel les points du premier seront remplacés par des droites et les droites par des points. En géometrie à trois dimensions, lorsqu'un théorème indépendant de toutes relations métriques d'angles et de longueurs se trouve démontré, on peut en conclure immédiatement, et sans qu'il soit besoin de demonstration, un autre théorème, dans lequel les points du premier seront remplacés par des plans et les plans par des points, les droites restant en même nombre dans les deux théorèmes."

En Gergonne es bien claro que el principio está determinado por la 
independencia de propiedades métricas e implícitamente por la introducción de elementos impropios.

Con todo debemos señalar otros dos aspectos que ya están en Gergonne. En primer lugar la creciente importancia de la deducción en el uso del principio, por más que sea aún remota una presentación axiomática que permita dar a la dualidad su lugar sistemático. Si bien en sus escritos metodológicos (1809) ya daba una idea clara de la construcción de los sistemas deductivos, Gergonne no se molesta en disponer sus proposiciones, en geometría proyectiva, de una manera sistemática estricta de acuerdo con ellos. Con todo, en sus Considerations... Gergonne procede a partir de "Nociones primitivas" hacia teoremas de cierto grado de complicación. Gergonne ve muy claro - cosa acorde con el ideal metódico citado de Poncelet-, que no se trata de lograr una cosecha de teoremas aislados; el principio de dualidad da entonces una herramienta metódica para ese objetivo.

En segundo lugar, y esto es ya claro desde Gergonne, pero se va a acentuar en las sucesivas versiones de la dualidad que fueron apareciendo por lo menos hasta el fin del siglo, los enunciados que se dan del principio incluyen, nada veladamente, consideraciones metamatemáticas, u otras, que adquieren, en algunos casos, un carácter metamatemático indudable.

Sabemos bien que sólo a partir de cierto momento y especialmente a partir de sistemas axiomáticos de tendencia formalizante se plantean problemas metamatemáticos en forma rigurosa y generalizada, en especial los de consistencia y completitud. Pero ya algunas formas de enunciar el principio de dualidad se refieren a conjuntos de teoremas como tema: "si es demostrable el conjunto $\mathbf{C}$ de teoremas, es demostrable el conjunto $\mathrm{C}^{\prime}$ de duales de los de $\mathrm{C}$; si $\mathrm{A}$ es un axioma, su dual también lo es; si se posee una demostración de un teorema $T$, conformada por un conjunto de pasos, la demostración del dual $\mathrm{T}^{\prime}$ estañá formado por pasos que son respeotivamente los duales de aquéllos".

Todo esto está dicho ya en Gergonne y si su "metamatemática" es apenas un esbozo respecto a las que conocemos hoy, de todas maneras no se limita a la utilización de términos en el metalenguaje sino que procede a considerar la deducción en su conjunto, y en sus pasos, de ese modo. El carácter metamatemático pre-axiomático está bien definido y da todo lo que puede dar en esa etapa.

Pero es necesario ver además en qué sentido el principio de dualidad marca el abandono de una geometría física, dentro de un proceso complejo pero indudable hacia la construcción de una geometría abstracta.

4.4.1. La tendencia a buscar métodos aún en geometría sintética, la creación de conceptos (elementos impropios) y de un lenguaje también 
nuevo, con sus virtualidades, la utilización de un principio, entre otros, de dualidad, con las características señaladas, son aspectos complementados por el desarrollo no-métrico, por parte de von Staudt, de una geometría proyectiva que ha sido llamado purista; todo ello en el periodo que se cierra en 1847, y configura en su conjunto una producción matemática notable. Además, si como hemos dicho, la geometría proyectiva tenía una vocación no-métrica, y de hecho recogía las propiedades nométricas, sin embargo la intervención de la relación inarmónica iba en contra de esa tendencia. Por ello cuando von Staudt da un procedimiento exclusivamente sintético, por medio de coordenadas homogéneas, de construir "la cuarta", se valida, de hecho, hacia atrás, toda la tendencia indicada. No hay de todos modos necesidad de atribuir a la geometría proyectiva anterior a von Staudt el carácter purista que con él adquiere, porque los otros elementos que le pertenecen configuran ya un logro de magnitud insoslayable. Entonces de 1822 a 1847 se asiste a un desarrollo de la geometría proyectiva, algunos de cuyos elementos hemos apuntado, que no tiene igual.

Se ha afimado (Lolli) que, con los Grundlagen der Geometrie, Hilbert dio, por lo menos para la geometría, la sanción oficial a la transformación de la matemática en lo que ha sido desde entonces. Démoslo por supuesto.

Por otra parte, es un lugar común el afirmar la centralidad de las geometrias no euclidianas en el proceso de esa transformación. Como consecuencia de las exageraciones en este sentido se ha llegado a decir, con toda razón, que las geometrías no euclidianas son la tarte à la crème des épistémologues.

Lejos de desconocer el aporte singular que ellas presentan, argumentamos aquí contra esa centralidad.

5. Los caracteres que hemos señalado para la geometría proyectiva en el periodo considerado (preferencia por un enfoque metódico aun en su faz sintética, introducción elaborada de elementos impropios, estudio de propiedades no-métricas, uso amplio de la dualidad) la sitúan en un lugar destacado del desarrollo de la geometría que culminaría con Hilbert-99. Si bien la geometría proyectiva pertenece al periodo pre-axiomático (por más que luego $M$. Pasch haya formulado para ella una de las primeras axiomáticas modernas), se esbozan en su elaboración formas de producir geometría totalmente originales. Esto se produce de manera temprana y con una continuidad que no se da en otros casos.

Como hemos apuntado se trata de una geometría no física en el sentido en que eran físicas tanto la clásica euclídea como la geometría analítica; además, el principio de dualidad hace que su desarrollo estructu- 
ral sea tan significativo como el de su contenido ya de por sí diferente del de aquéllas.

De un modo peculiar, es cierto, podemos decir de la geometría proyectiva que es una geometría no euclídea. Y que, antes que las de Lobatchevsky, Bolyai y Riemann, rompe (de modo incruento) con una tradición milenaria. Por más que el estudio de las cónicas haya estado en sus origenes más remotos, la geometría proyectiva alcanza luego una generalidad inusual.

La propia presentación de Lobatchevsky y su conceptuación, sin contar con la filosofía empirista que él defendía, tenían en principio un correlato físico; y su elaboración posterior se hizo en el marco de la relación entre geometría y espacio físico. La geometría proyectiva, mucho antes, ya no era una teoría de la extensión ni del espacio. Aunque esto no sea explícito debemos recordar que sólo muy tardíamente se reconoce una separación entre geometría y teoría del espacio y muchas de las discusiones de fin de siglo se producen porque muy trabajosamente se llega a este reconocimiento.

Una geometría en la que punto y recta son sustituibles por recta y punto, respectivamente, pone de hecho, aunque no explícitamente, su mirada hacia las relaciones entre entes no fijos (más aún si pensamos que en la geometría proyectiva de tres dimensiones la intercambiabilidad es otra). ¿Debemos pensar como secundaria esa intercambiabilidad?, ¿o más bien pensar que (aun con las excepciones que señalamos) no hay al comienzo una conciencia totalmente clara de ella? ¿Por qué no aparece como revolucionaria una geometría de este tipo mientras que inmediatamente aparecen como tales las geometrías no euclídeas? ¿Por qué hay una resistencia - bien documentada - contra éstas y escasa contra la geometría proyectiva? (el caso de Descartes es de tomarse en cuenta). La respuesta no es fácil y no haremos más que sugerir, de modo nada novedoso, algunas circunstancias vinculadas a las características respectivas ya señaladas.

En primer lugar las geometrías no-euclídeas rompen radicalmente con una tradición geométrica (en geometría plana), consolidada y vinculada a creencias cientificas, y no científicas, insertas en la concepción del mundo dominante y en filosofías también dominantes. $Y$ rompe en forma bien localizada, respecto al postulado quinto, fácilmente focalizable aun con consecuencias más amplias. En segundo lugar esto mismo; esa focalización es factible y para nada exótica porque se trata de un tema trabajado (el del postulado V), enseñado aún en el nivel elemental, y accesible por tanto a estudiosos con relativamente escasa formación geométrica. Más aún, la ruptura es notoria porque se trata de un tema con claras implicaciones físicas. Que los beocios fueran sobre todo filósofos 
poco atentos al desarrollo científico real tiene algo que ver con estas características del punto de ruptura.

Por el contrario, la revolución tranquila, enmascarada, que aporta la geometría proyectiva, y cuyas características hemos tratado de esbozar, tiene que ver con un complejo cuerpo de doctrina, accesible, no sólo pero particularmente, a especialistas. El corte con la geometría tradicional no es puntual, ni siquiera localizado, como el del caso comparado, sino que involucra la construcción total de la geometría, o de amplias partes de ella; involucra también una nueva concepción de los entes geométricos a la luz de sus relaciones. El choque no es aparente, no se hace aparente, y por ello mismo el enmascaramiento de la ruptura se hace factible. Por otra parte, sólo la integración, debida fundamentalmente a Klein, permite, ya en otro momento, ver con claridad relaciones interteóricas que iluminan a su vez a cada una de las geometrías puestas en relación y en particular el caso de la geometría proyectiva, de modo desfasado en el tiempo.

Hemos querido apenas esbozar algunas propuestas de explicación sin elaborarlas más. Hemos querido señalar como sintomático el hecho de que de 1822 a 1847 (y aún hasta 1867, fecha clave) la geometría proyectiva adquirió un grado de madurez y de elaboración significativos, acentuó gradualmente, de Poncelet a von Staudt, el carácter abstracto que va a dominar de modo creciente a la geometría, culminando con Hilbert. 